\title{
Poster abstract: The ZotBins solution to Waste Management using Internet of Things
}

\author{
Joshua Cao, Jesse Chong, Marissa Lafreniere, Owen Yang, Primal Pappachan, Sharad Mehrotra, \\ Nalini Venkatasubramanian \\ University of California, Irvine \\ \{caoj11,chongjl,mlafreni,okyang\}@uci.edu \\ \{primal,sharad,nalini\}@ics.uci.edu
}

\begin{abstract}
The growing use of Internet of Things (IoT) technologies has the potential to improve waste management. In this work, we present ZotBins, a system that facilitates sustainable practices for individuals and improves waste management efficiency for organizations. ZotBins consists of smart bins fitted with sensors, a waste recognition framework, and a variety of user applications. Currently, eight smart bins have been deployed in University of California, Irvine (UCI) campus and the preliminary estimates show that it improves the diversion rate (percentage of waste diverted from landfills through methods such as recycling) by about $20 \%$ while generating tens of thousands of dollars in revenue over the course of next ten years for the university.
\end{abstract}

\section{CCS CONCEPTS}

- Hardware $\rightarrow$ Sensor applications and deployments; • Applied computing;

\section{KEYWORDS}

Internet of Things, Waste Management, User Applications

\section{ACM Reference Format:}

Joshua Cao, Jesse Chong, Marissa Lafreniere, Owen Yang, Primal Pappachan, Sharad Mehrotra, Nalini Venkatasubramanian . 2020. Poster abstract: The ZotBins solution to Waste Management using Internet of Things. In The 18th ACM Conference on Embedded Networked Sensor Systems (SenSys '20), November 16-19, 2020, Virtual Event, Japan. ACM, New York, NY, USA 2 pages. https://doi.org/10.1145/3384419.3430441

\section{INTRODUCTION}

Waste poses significant economic, environmental, and public health threats if not addressed properly. At the current rate where $64.8 \%$ of waste (specifically municipal solid waste) is being sent to landfills, the economic opportunity to reuse materials is lost, greenhouse gas emissions are produced, and air and water quality are compromised for certain communities [1]. To maximize the diversion rate, three critical challenges must be solved: 1) in-depth analysis of waste trends [5]; 2) maximizing material reuse potential through waste recognition [2]; 3) reduction of facilities management overhead [3]

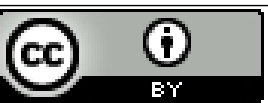

This work is licensed under a Creative Commons Attribution International 4.0 License. SenSys '20, November 16-19, 2020, Virtual Event, Japan

(c) 2020 Copyright held by the owner/author(s)

ACM ISBN 978-1-4503-7590-0/20/11...\$15.00

https://doi.org/10.1145/3384419.3430441
ZotBins ${ }^{1}$ is a holistic IoT based solution that provides many useful features to assist organizations (e.g., facilities management) and general users in maximizing diversion rate by addressing these challenges.

ZotBins consists of smart bins fitted with a suite of sensors that monitor data about various waste events. ZotBins helps organizations analyze this data using different visualizations to study real-time and historical diversion rates and to determine appropriate strategies for waste pickup and bin placement. It also includes different user applications that guide users to appropriately dispose of their waste. In this work, we present a detailed overview of ZotBins and describe the current deployment status of ZotBins on UCI campus.

\section{ZOTBINS SYSTEM}

ZotBins consists of the following three modules. First, the Sensors and Controller are attached to the waste bin and are used for the collection of sensor data and the sending of collected data to TIPPERS[4] through API calls. TIPPERS is a cloud based IoT data management system that is used to process and store all sensor data. Second, the Waste Recognition Framework identifies images or barcode numbers of waste from the smart bin or user applications. Third, User Applications, built on top of TIPPERS APIs, allows facilities management and general users to utilize various useful features. The data flow through these different modules in ZotBins is demonstrated in Figure 1.

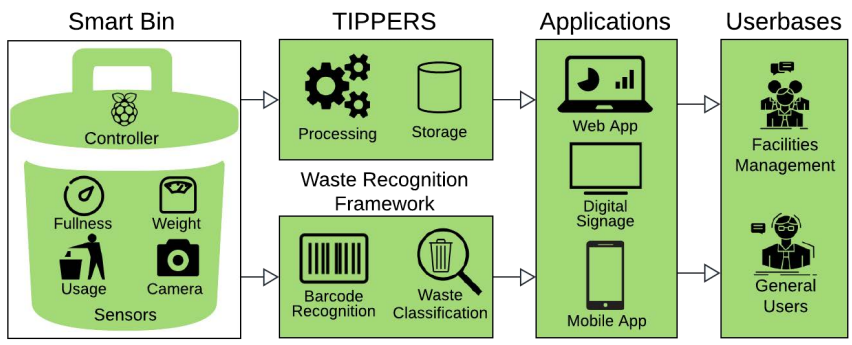

Figure 1: ZotBins System Data Flow

\section{Sensors and Controller}

Smart bins consists of the following sensors: 1) Weight (implemented using a load cell) measures the total weight of waste inside a bin, 2) fullness (implemented using the ultrasonic sensor) determines the percentage fullness of the bin, 3) usage (implemented using the break beam sensor) detects every time a bin is used, and 4) camera captures images of waste thrown away which is later used

\footnotetext{
${ }^{1}$ https://zotbins.github.io/
} 
as training data for the Waste Recognition Framework. Together these sensors log important statistics such as bin usage frequency, diversion rates, and fullness rates. The controller (implemented using a Raspberry Pi v3) is responsible for scheduling sensor data collection, sending it in batches to TIPPERS, and for logging any errors with sensors on the smart bin for maintenance purposes.

\section{Waste Recognition Framework}

Identifying waste from images has numerous use cases such as catering disposal guidance for users, automating waste audits for facilities management, or providing immediate feedback for users after they dispose their waste. Therefore we are developing a Waste Recognition Framework which recognizes waste using: 1) barcode number and 2) image recognition. The barcode recognition model identifies waste items by looking up the barcode number in a database of barcodes and returning the item type. For image recognition, we use a convolutional neural network that classifies single waste items in images into item types. After one of these models identifies a waste item's type, the Waste Recognition Framework searches for the corresponding disposal instructions from its database. Disposal instructions are input into the database by ZotBins members, or by general users through the User Applications. We currently have about 2,700 barcodes and 5,000 images stored in the Waste Recognition Framework database. This data is sourced primarily from external websites and databases.

\section{User Applications}

We developed three applications based on discussions from sustainability organizations at UCI. The first application is a web app focused on supporting facilities management containing two features: ZAnalytics and ZMap (shown in Figure 2). ZAnalytics allows facilities management to perform historical analysis of sensor data by displaying graphs and trends for bins over a selected time period (last 6 months, last week, etc). ZMap is a feature for facilities management to gain insight into deployed bin locations and statuses. The user can zoom into a building to view its floor plans with icons representing where bins are located. After selecting an individual bin, the user can view bin logs, data trends, and a snapshot of the bin's waste content.

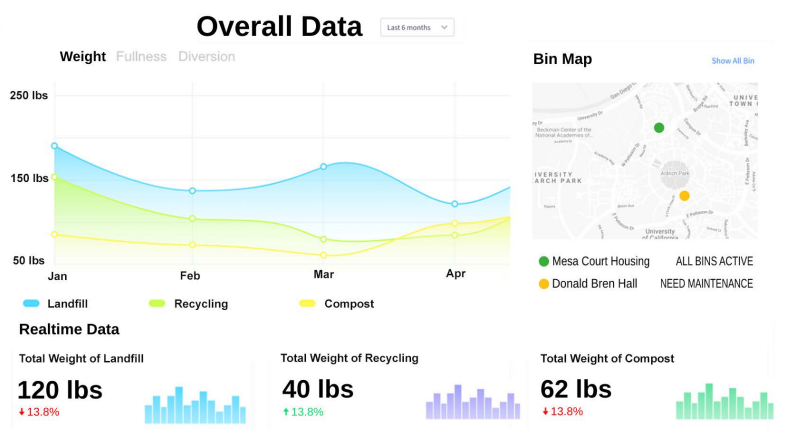

Figure 2: Web App displaying ZAnalytics and ZMap

The second application is a mobile app focused on supporting general users with waste disposal guidance. The mobile app provides a feature called ZBuddy in which a user can receive waste disposal instructions after submitting a picture or barcode of their trash. ZBuddy will also display the nearest relevant bin location on a map along with disposal instructions for the identified waste. Our third application is a digital signage connected to the controller and mounted on the waste bins. This digital signage displays animated pop-ups of educational content whenever a waste item is thrown away and proper disposal instructions. The digital signage is shown deployed in Figure 3.

\section{DEPLOYMENT AND FUTURE WORK}

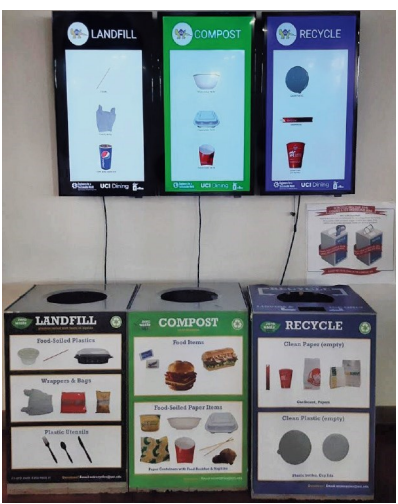

Figure 3: Smart Bin Set with Digital Signage
Since June 2019, we have been collaborating with UCI Dining and have deployed eight smart bins in campus cafeterias and department buildings (Figure 3). All these bins have weight and ultrasonic sensors installed from which we have collected more than 100,000 data points. Additionally, some of these bins were fitted with break beam sensors and cameras from which we have collected another 13,000 frequency data points and 150 images. By analyzing the break beam data, we found that the ZotBins deployed in cafeterias are used 437 times on average per weekday. Digital signage has been installed on two smart bin sets (a set includes three bins: recycling, compost, and landfill) to aid UCI Dining's educational outreach goal. We compared the effectiveness of a smart bin set with digital signage against a set of regular bins using our deployment. Over a period of 3 months, we recorded increase in composting (22.72\%) and recycling (5.53\%) rates compared to the regular bins. Converting these rates to their monetary values, we found that the smart bin set with digital signage saves $\$ 20,602.29$ for facilities management over a period of 10 years ${ }^{2}$.

As illustrated, ZotBins provides significant environmental and economic benefits to communities. We are currently working on improving and testing the accuracy of the Waste Recognition Framework, adding more features to user applications, and mass producing smart bins for the entire campus. We are also working on open sourcing ${ }^{3}$ ZotBin's code base and hardware schematics so that other developers can expand upon our work to bring positive impacts to their communities.

\section{REFERENCES}

[1] 2017. National Overview: Facts and Figures on Materials, Wastes and Recycling. (2017). https://www.epa.gov/facts-and-figures-about-materials-wasteand-recycling/national-overview-facts-and-figures-materials

[2] Chu et al. 2018. Multilayer hybrid deep-learning method for waste classification and recycling. Computational Intelligence and Neuroscience 2018 (2018).

[3] Faye et al. 2019. No Time to Waste, with SWAM!. In BuildSys. 342-343.

[4] Mehrotra et al. 2016. TIPPERS: A privacy cognizant IoT environment. In 2016 PerCom Workshops. IEEE, 1-6.

[5] Zaman et al. 2013. The zero waste index: a performance measurement tool for waste management systems in a 'zero waste city'. F. Clean. Prod. 50 (2013), 123-132.

\footnotetext{
${ }^{2}$ More details of cost-benefit analysis can be read at https://sites.uci.edu/zotbins/2020/ $09 / 20$ /cost-benefit-analysis-of-zotbins/

${ }^{3}$ https://github.com/zotbins/
} 